\title{
Utilization of Membranous Lipid Substrates by Membranous Enzymes
}

\author{
HYDROLYSIS OF SPHINGOMYELIN IN ERYTHROCYTE 'GHOSTS' AND LIPOSOMES BY \\ THE MEMBRANOUS SPHINGOMYELINASE OF CHICKEN ERYTHROCYTE 'GHOSTS'
}

\author{
Michel RECORD, ${ }^{*} \dagger$ Abraham LOYTER $\ddagger$ and Shimon GATT* \\ *Laboratory of Neurochemistry, Department of Biochemistry, Hebrew University-Hadassah \\ Medical School, Jerusalem, Israel, and $\ddagger$ Department of Biological Chemistry, Institute of Life Sciences, \\ The Hebrew University of Jerusalem, Jerusalem, Israel
}

(Received 3 July 1979)

\begin{abstract}
Incubation at $37^{\circ} \mathrm{C}$ of haemolysed chicken erythrocytes ('chicken erythrocyte ghosts') resulted in hydrolysis of the membrane sphingomyelin, suggesting an activation of a latent sphingomyelinase during the haemolysis procedure. When this incubation was continued for several hours, the entire sphingomyelin of the erythrocyte 'ghosts' was hydrolysed and membranes were obtained that were devoid of sphingomyelin, but had an active sphingomyelinase. Mixing the latter membranes with human erythrocyte 'ghosts' or positively charged liposomes led to hydrolysis of the sphingomyelin in these two membranes. This suggested that, after haemolysis, the activated sphingomyelinase in the membrane of the chicken erythrocyte 'ghosts' could hydrolyse sphingomyelin in its own membrane ('intramembrane utilization') or adjacent membranes ('intermembrane utilization').
\end{abstract}

Most enzymic studies with lipids have used aqueous dispersions of the substrates and membranous enzymes or partially purified preparations extracted from membranes (Gatt, 1973; Gatt \& Barenholz, 1973). In biological systems most lipids are integral components of membranes, and the enzymes utilizing them are also membrane bound. In spite of extensive studies (Gatt, 1973; Gatt \& Barenholz, 1973; Gatt et al., 1978), the mode of utilization of lipids by their respective enzymes in biological membranes has yet to be elucidated.

In previous papers the interaction of a membranous substrate (diacylglycerol) with a membranous enzyme [lipase of rat brain microsomal preparation (Cabot \& Gatt, 1976)] was investigated (Cabot \& Gatt, 1977; Rousseau \& Gatt, 1979). It was shown that the enzyme could degrade the substrate in its own as well as in adjacent membranes in which the enzyme had been inactivated (Rousseau \& Gatt, 1979). In another study it was shown that the sphingomyelin of chicken erythrocyte 'ghosts' was hydrolysed by a membranous sphingomyelinase after cell lysis (Hirshfeld \& Loyter, 1975). This system has now been used to

† Present address: Institute National de la Santé et de la Recherche Medicale, Unité de Recherches 101, Hôpital Purpan, 31052 Toulouse, France. investigate intramembrane and intermembrane utilization of this lipid by the membranous enzyme. This was done by analysing the kinetics of action of the chicken erythrocyte sphingomyelinase on sphingomyelin in its own membrane or in other membranes such as human erythrocyte 'ghosts'; the latter have sphingomyelin but no sphingomyelinase. The hydrolysis of sphingomyelin in a liposomal dispersion by the membranous enzyme was also investigated. A preliminary report of this work has already appeared in abstract form (Record \& Gatt, 1979).

\section{Experimental Procedure}

\section{Preparation of chicken erythrocyte 'ghosts'}

Chicken erythrocytes were washed with Solution $\mathrm{K}\left(135 \mathrm{~mm}-\mathrm{KCl}, 5.4 \mathrm{~mm}-\mathrm{NaCl}, 1 \mathrm{~mm}-\mathrm{MgSO}_{4}\right.$ and $25 \mathrm{~mm}$-Tricine $\{N$-[2-hydroxy-1,1-bis(hydroxymethyl)ethyl]glycine $\} / \mathrm{NaOH}, \mathrm{pH}$ 7.4) as previously described (Toister \& Loyter, 1975). Aged human erythrocytes, types $\mathrm{A}^{+}$, were obtained from the blood bank of Hadassah Hospital, Jerusalem, and washed with Solution K, as described (Toister \& Loyter, 1973). The erythrocytes were used within 5 days after washing. Human or chicken erythrocytes were haemolysed by suspending a pellet of washed 
cells in 10-20vol. of a hypo-osmotic medium containing $25 \mathrm{~mm}$-Tricine/NaOH, pH 7.4. After $10 \mathrm{~min}$ at $4^{\circ} \mathrm{C}$, the haemolysed cells were mixed with an equal volume of the following media: (1) $2 \mathrm{mM}-\mathrm{MgCl}_{2}$ in $25 \mathrm{~mm}$-Tricine $/ \mathrm{NaOH}, \mathrm{pH} 7.4$, yielding a hypo-osmotic medium; (2) $0.3 \mathrm{M}-\mathrm{NaCl}$, containing $25 \mathrm{~mm}$-Tricine/ $\mathrm{NaOH}, \mathrm{pH} 7.4$, and $2 \mathrm{~mm}$ $\mathrm{MgCl}_{2}$, yielding an iso-osmotic medium; (3) $66 \%$ glycerol, containing $25 \mathrm{~mm}$-Tricine/ $\mathrm{NaOH}, \mathrm{pH} 7.4$, and $2 \mathrm{mM}-\mathrm{MgCl}_{2}$, yielding a hyper-osmotic medium. For the preparation of enucleated chicken erythrocyte 'ghosts', a pellet of washed erythrocytes was suspended in 5 vol. of a hypo-osmotic solution containing $25 \mathrm{~mm}-\mathrm{NaCl}$ and $25 \mathrm{~mm}$-Tricine $/ \mathrm{NaOH}$, pH 7.4. After homogenization (20 strokes in a Dounce homogenizer), the haemolysed cells were centrifuged at $1000 \mathrm{~g}$ for $5 \mathrm{~min}$ in the cold. The supernatant contained the enucleated chicken erythrocyte 'ghosts', and the pellet contained intact erythrocytes, nucleated erythrocyte 'ghosts' and aggregated nuclei.

\section{Estimation of sphingomyelin hydrolysis}

Chicken erythrocyte 'ghosts' were suspended in one of the above described media, to give a concentration of $5-10 \%(\mathrm{v} / \mathrm{v})$. Incubation was done at $37^{\circ} \mathrm{C}$ in volumes of $2 \mathrm{ml}$, by themselves or with equal volumes of human erythrocyte 'ghosts'. During the incubation, the sphingomyelin of these membranes was hydrolysed by a sphingomyelinase located in the chicken erythrocyte 'ghosts' (Hirshfeld \& Loyter, 1975). The reaction was terminated by the addition of $4 \mathrm{ml}$ of methanol and lipids were extracted by further addition of $2 \mathrm{ml}$ of chloroform. To facilitate the extraction, the resulting suspension was heated for $10 \mathrm{~min}$ at $50^{\circ} \mathrm{C}$. For a quantitative estimation of the recovery of lipids, $\left[{ }^{14} \mathrm{C}\right]$ cholesterol (about 20000d.p.m.) was added as an internal control. After removing the denatured proteins by centrifugation, $2 \mathrm{ml}$ each of the chloroform and water were added to the extract. The upper aqueous-methanolic phase was discarded and the lower chloroform phase was evaporated at 70$80^{\circ} \mathrm{C}$. To improve the resolution of the sphingomyelin and to avoid its overlapping with other phospholipids during t.l.c., ester bonds of glycerophospholipids were hydrolysed by the addition of $0.5 \mathrm{ml}$ of $1 \mathrm{M}-\mathrm{KOH}$ in methanol to the dried residue, followed by incubation for $1 \mathrm{~h}$ at $37^{\circ} \mathrm{C}$. Hydrolysis was stopped by the addition of $1 \mathrm{ml}$ of chloroform/water $(1: 1, \mathrm{v} / \mathrm{v})$. After discarding the upper phase, the lower phase was washed with $2 \mathrm{vol}$. of methanol/water $(1: 1, \mathrm{v} / \mathrm{v})$, dried, dissolved in about $60 \mu \mathrm{l}$ of chloroform/methanol $(1: 1, \mathrm{v} / \mathrm{v})$ and applied to thin-layer plates of silica gel HR $(0.25 \mathrm{~mm})$, which were developed in chloroform/ methanol/water (65:25:4, by vol.). After identifying the phospholipids by $I_{2}$ vapour, the spots corresponding to a marker of authentic sphingomyelin were scraped, the silica was ashed and the phosphorus content was determined by the method of Bartlett (1959). The yield of sphingomyelin was estimated by the recovery from the plate of $\left[{ }^{14} \mathrm{C}\right]$ cholesterol, which was counted for radioactivity by using $2 \mathrm{ml}$ of a mixture of Triton $\mathrm{X}-100 /$ ethanol $(1: 1, \mathrm{v} / \mathrm{v})$, to which $10 \mathrm{ml}$ of toluene scintillation liquid was added.

\section{Depletion of sphingomyelin of chicken erythrocyte 'ghosts'}

Chicken erythrocyte 'ghosts', nucleated or enucleated, were suspended in the hyperosmotic medium (see above) and incubated for about $15 \mathrm{~h}$ at $37^{\circ} \mathrm{C}$ with gentle shaking. This led to the hydrolysis of about $95 \%$ of the membrane sphingomyelin. The sphingomyelin-depleted erythrocyte 'ghosts' were collected by centrifugation, at $4^{\circ} \mathrm{C}$, for $10-15 \mathrm{~min}$ at $5000 \mathrm{~g}$, using the nucleated and at $50000 \mathrm{~g}$ using the enucleated preparations.

Hydrolysis of dispersions of sphingomyelin by sphingomyelinase of chicken erythrocyte 'ghosts'

(a) Mixed micelles of sphingomyelin and Triton $X-100$. [ $\left.{ }^{3} \mathrm{H}\right] \mathrm{Choline-labelled} \mathrm{sphingomyelin} \mathrm{was} \mathrm{pre-}$ pared as previously described (Stoffel, 1975; Gatt, 1976). The reaction mixture contained, in a final volume of $0.2 \mathrm{ml}$, the following ingredients: $60 \mathrm{nmol}$ of $\left[{ }^{3} \mathrm{H}\right]$ sphingomyelin (about 65000 d.p.m.), $0.2 \mathrm{mg}$ of Triton X-100, $0.2 \mu \mathrm{mol}$ of $\mathrm{MgCl}_{2}, 5 \mu \mathrm{mol}$ of Tricine $/ \mathrm{NaOH}, \mathrm{pH} 7.4$, and about $0.1 \mathrm{mg}$ of chicken erythrocyte 'ghosts'. After incubation at $37^{\circ} \mathrm{C}$, the reaction was terminated by the addition of $0.8 \mathrm{ml}$ of chloroform/methanol, $(2: 1, \mathrm{v} / \mathrm{v})$. The radioactivity of $\left[{ }^{3} \mathrm{H}\right]$ choline in $0.2 \mathrm{ml}$ of the upper phase was determined after adding $2.8 \mathrm{ml}$ of Instagel (Packard).

(b) Sphingomyelin in liposomes. The reaction mixture contained, in a final volume of $0.3 \mathrm{ml}$, the following: $60 \mathrm{nmol}$ of $\left[{ }^{3} \mathrm{H}\right]$ sphingomyelin incorporated in unilamellar liposomes (see the procedure in the following paragraph), $0.3 \mu \mathrm{mol}$ of $\mathrm{MgCl}_{2}$, $7.5 \mu \mathrm{mol}$ of Tricine $/ \mathrm{NaOH}, \mathrm{pH} 7.4$, and $3.8 \mathrm{mg}$ of chicken erythrocyte 'ghosts'. After incubation at $37^{\circ} \mathrm{C}$, the reaction was terminated as above, and $0.3 \mathrm{ml}$ of the upper phase was counted for radioactivity in a mixture containing $3 \mathrm{ml}$ of Triton X-100 and $7 \mathrm{ml}$ of toluene-based scintillation fluid.

\section{Preparation of liposomes}

Liposomes containing sphingomyelin or phosphatidylcholine were prepared essentially by the procedure of Barenholz et al. (1977). To prepare liposomes containing more than one lipid, chloroform/methanol solutions of hexadecylamine or dicetyl phosphate were mixed with sphingomyelin or phosphatidylcholine. The solvent was evaporated under $\mathrm{N}_{2}$, buffer was added and the suspension was 
subjected to ultrasound. The sonicated dispersions were centrifuged at room temperature for $10 \mathrm{~min}$ at $3000 \mathrm{rev} . / \mathrm{min}$ and the supernatant was retained. For the adsorption experiments, the ultrasonicated dispersions were centrifuged for $30 \mathrm{~min}$ at $180000 \mathrm{~g}$ and the supernatant was retained.

\section{Materials}

Sphingomyelin and egg phosphatidylcholine were purchased from Makor Chemicals, Jerusalem. Dicetyl phosphate was from Sigma and hexadecylamine was from Fluka. Protein was determined by the procedure of Lowry et al. (1951), adding sodium dodecyl sulphate to the tartrate solution (final concentration $0.07 \%, w / v)$.

\section{Results}

Hydrolysis of sphingomyelin in chicken erythrocyte 'ghosts'

Sphingomyelin of chicken erythrocyte membranes is hydrolysed when the erythrocytes are haemolysed for several minutes in a hypo-osmotic medium (Hirshfeld \& Loyter, 1975). Fig. 1 (upper

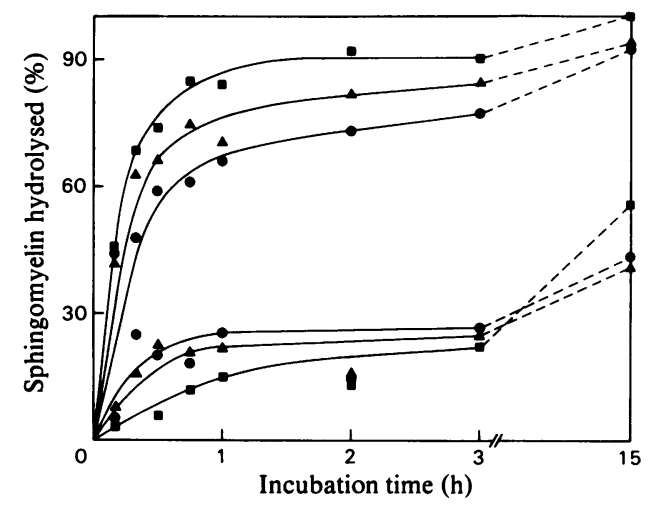

Fig. 1. Dependence of sphingomyelin hydrolysis in chicken or human erythrocyte 'ghosts' on incubation time

Erythrocytes were lysed and diluted with the respective three media, as described below (see also the Experimental Procedure section). The three upper curves describe the results obtained by incubating only chicken erythrocyte 'ghosts'. The three lower curves describe the results obtained by using mixtures containing equal amounts of sphingomyelin-depleted chicken erythrocyte 'ghosts' and freshly lysed human erythrocyte 'ghosts' (4 mg each). Incubation was performed in the following media: 0 , hypo-osmotic medium ( $1 \mathrm{mM}-\mathrm{MgCl}_{2}$, $25 \mathrm{~mm}$-Tricine/ $\mathrm{NaOH}, \quad \mathrm{pH} 7.4) ; \quad \Delta$, iso-osmotic medium $\quad(0.15 \mathrm{M}-\mathrm{NaCl}, \quad 25 \mathrm{mM}-\mathrm{Tricine} / \mathrm{NaOH}$, pH 7.4, $1 \mathrm{~mm}-\mathrm{MgCl}_{2}$ ); $\mathbf{\square}$, hyper-osmotic medium [33\% glycerol (v/v), $25 \mathrm{~mm}$-Tricine/ $\mathrm{NaOH}, \mathrm{pH} 7.4$, $1 \mathrm{~mm}-\mathrm{MgCl}_{2}$ ]. three curves) shows the rate of hydrolysis of sphingomyelin in the membranes of chicken erythrocyte 'ghosts'. The cells were lysed and incubated in three media of various tonicities (see the Experimental Procedure section). As can be seen in Fig. 1, the rates of hydrolysis were similar in the three respective media. The data of the top three curves in Fig. 1 demonstrate a very rapid degradation of sphingomyelin, reaching up to $50 \%$ or more in $10 \mathrm{~min}$, and more than $80 \%$ in $30 \mathrm{~min}$ of incubation at $37^{\circ} \mathrm{C}$.

Fig. 2 presents data of an experiment similar to that of the upper curve of Fig. 1, showing the concentration of the remaining unhydrolysed sphingomyelin in chicken erythrocyte 'ghosts' as a function of time. In this experiment a fixed concentration of membranes was used and the changes in sphingomyelin concentration were a consequence of the intramembrane enzymic hydrolysis of this phospholipid. The curve describing this relationship was a rectangular hyperbola (Fig. $2 a$; also see upper inset for the reciprocal presentation, Fig. $2 b$ ). The lower inset (Fig. 2c) demonstrates that the curve describing the rate of hydrolysis of sphingomyelin as a function of the concentration of this lipid in the chicken erythrocyte membranes was parabolic.

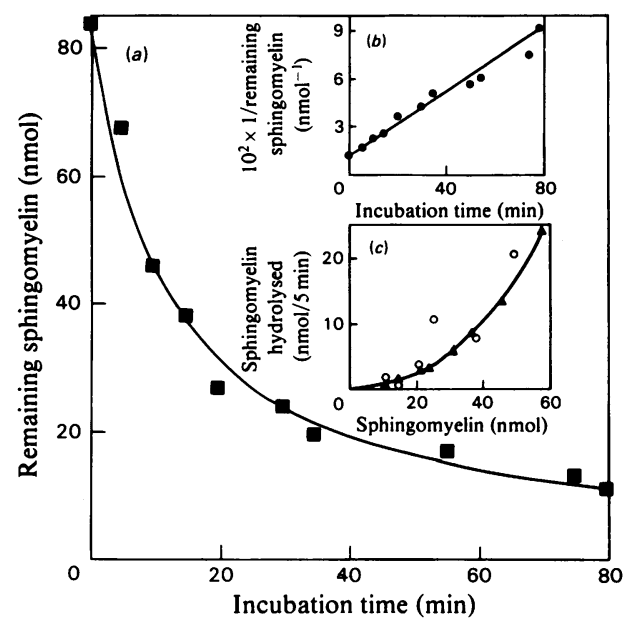

Fig. 2. Hydrolysis of chicken erythrocyte sphingomyelin as a function of incubation time and substrate concentration

(a) The data were obtained from an experiment similar to that described by the upper curve of Fig. 1 , namely, hydrolysis of sphingomyelin of chicken erythrocyte 'ghosts' incubated in the hyperosmotic medium; (b) shows a reciprocal presentation of the data of Fig. $2(a) ;(c)$ shows the rate of hydrolysis as function of sphingomyelin concentration in chicken erythrocyte 'ghosts'. $\Delta$, data calculated from curve in Fig. 2(a); O, data obtained from a separate experiment. 
Hydrolysis of sphingomyelin in human erythrocyte 'ghosts' by chicken erythrocyte sphingomyelinase

The very fast hydrolysis shown in the upper three curves of Fig. 1 suggested that sphingomyelin in chicken erythrocyte 'ghosts' was hydrolysed by the enzyme residing in the same membranes. This can therefore be classified as an intramembrane utilization (for a second system see Rousseau \& Gatt, 1979). To examine whether an intermembrane interaction and utilization also exists, chicken erythrocyte 'ghosts' were depleted of their sphingomyelin by overnight incubation and the mixed with human erythrocyte 'ghosts'; the latter contain sphingomyelin but no sphingomyelinase (Hirshfeld \& Loyter, 1975). The results of the lower three curves of Fig. 1 demonstrate that the sphingomyelin of the human erythrocyte 'ghosts' was indeed hydrolysed by the sphingomyelinase of the chicken erythrocyte 'ghosts', thus establishing the existence of an intermembrane interaction and substrate utilization. The relative rates of hydrolysis in the three media were similar, though in this case they seemed to be smaller for the hyper-osmotic medium. Comparison of the upper and lower curves of Fig. 1 shows that the rate as well as the degree of hydrolysis of sphingomyelin by the chicken erythrocyte enzyme were smaller in the intermembrane as compared with the intramembrane utilization.

The dependence of the enzymic activity, by the intermembrane route, on substrate concentration was studied by mixing increasing concentrations of human erythrocytes as source of substrate with a fixed amount of sphingomyelin-depleted enucleated chicken erythrocytes as source of enzyme (Fig. 3).

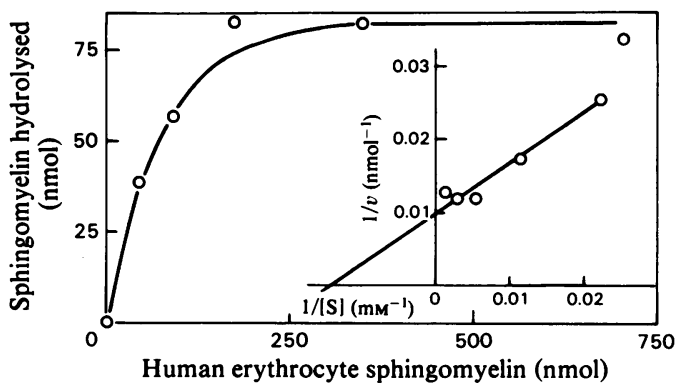

Fig. 3. Hydrolysis of sphingomyelin of human erythrocyte 'ghosts' by sphingomyelinase of chicken erythrocyte 'ghosts'

Incubation mixtures contained $10 \mathrm{mg}$ of enucleated sphingomyelin-depleted chicken erythrocyte 'ghosts', suspended in a final volume of $5 \mathrm{ml}$ of $25 \mathrm{~mm}$-Tricine, $\mathrm{pH} 7.4$, containing $25 \mathrm{~mm}-\mathrm{NaCl}$ and increasing concentrations of human erythrocyte 'ghosts' (expressed as sphingomyelin content). The incubation time was $2 \mathrm{~h}$.
The $v$-versus- $[\mathbf{S}\rfloor$ curve is of a substrate-saturation type; the maximal velocity of this intermembrane utilization was about $5 \mathrm{nmol}$ of sphingomyelin hydrolysed by $1 \mathrm{mg}$ of protein in $1 \mathrm{~h}$. In other experiments (results not shown) a fixed concentration of human erythrocyte 'ghosts', equivalent to $88 \mathrm{nmol}$ of sphingomyelin, was incubated with increasing concentrations of sphingomyelin-depleted enucleated chicken erythrocyte 'ghosts'. Use of the enucleated erythrocyte 'ghosts' resulted in linear and greater reaction rates than those shown in Fig. 1 in which nucleated preparation was employed. In a hypo-osmotic medium at $37^{\circ} \mathrm{C}$, up to $70 \%$ of the sphingomyelin of human erythrocyte 'ghosts' was hydrolysed during $2 \mathrm{~h}$ using $6 \mathrm{mg}$ of chicken erythrocyte membranes.

\section{Hydrolysis of sphingomyelin in liposomal dispersion}

Intermembrane utilization of sphingomyelin was studied by interacting unilamellar sealed vesicles of ${ }^{3} \mathrm{H}$-labelled sphingomyelin with sphingomyelin-depleted chicken erythrocyte 'ghosts'. As described previously (Gatt et al., 1973), three types of liposomes were prepared: neutral, negatively charged (by the addition of dicetyl phosphate) or positively charged (by the addition of hexadecylamine). Fig. 4 shows that the rate of hydrolysis of sphingomyelin in the uncharged (molar ratio equals zero) or the negatively charged liposomes by the enzymes in the chicken erythrocyte 'ghosts' was low. The presence of hexadecylamine in the liposomes markedly stimulated the reaction. In experiments for

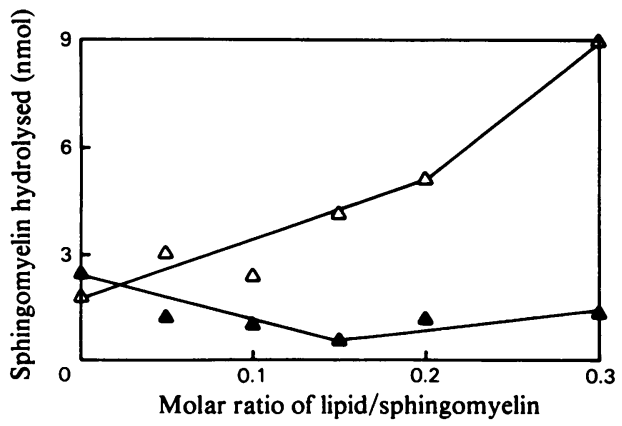

Fig. 4. Hydrolysis of $\left[{ }^{3} \mathrm{H}\right]$ sphingomyelin in liposomes containing increasing concentrations of dicetyl phosphate

$(\Delta)$ or hexadecylamine $(\Delta)$

$\left[{ }^{3} \mathrm{H}\right]$ Sphingomyelin and increasing concentrations of dicetyl phosphate or hexadecylamine were mixed, and liposomes were prepared (Barenholz et al., 1977). Incubation mixtures contained, in a final volume of $0.3 \mathrm{ml}, 3.8 \mathrm{mg}$ of sphingomyelin-depleted chicken erythrocyte 'ghosts' and $60 \mathrm{nmol}$ of liposomal $\left[{ }^{3} \mathrm{H}\right]$ sphingomyelin in $25 \mathrm{~mm}$-Tricine, pH 7.4, and $1 \mathrm{mM}-\mathrm{MgCl}_{2}$. The reaction was terminated after $1 \mathrm{~h}$ incubation at $37^{\circ} \mathrm{C}$. 


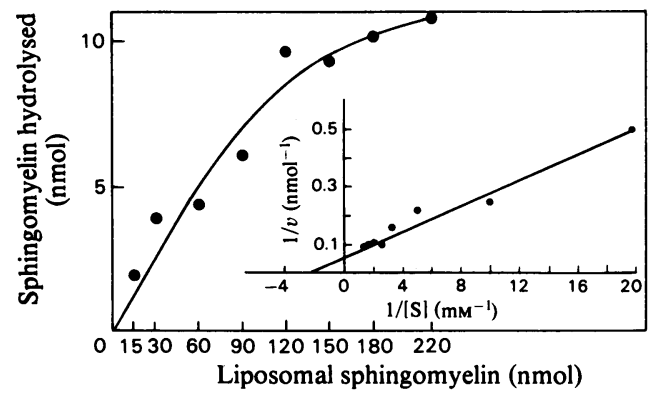

Fig. 5. Dependence of the rate of hydrolysis on the concentration of the liposomal sphingomyelin

Experimental conditions were as described in Fig. 4, except that increasing concentrations of liposomes, having a molar ratio of hexadecylamine to $\left[{ }^{3} \mathrm{H}\right]-$ sphingomyelin of 0.15 were used. Incubation time was $2 \mathrm{~h}$ at $37^{\circ} \mathrm{C}$.

which results are not shown, liposomes having $13 \mathrm{~mol} \%$ of hexadecylamine (molar ratio of amine to sphingomyelin $=0.15$ ) were incubated with sphingomyelin-depleted chicken erythrocyte 'ghosts'. The rate of sphingomyelin hydrolysis was directly proportional to incubation time (up to $1-2 \mathrm{~h}$ ). and protein concentration (up to about $3 \mathrm{mg}$ of protein). Fig. 5 shows that the curve describing the rate of hydrolysis as a function of increasing concentrations of the liposomal sphingomyelin was hyperbolic. The apparent $K_{\mathrm{m}}$ was $2.4 \mathrm{~mm}$ and $V_{\text {max. }}$ was $2.4 \mathrm{nmol} / \mathrm{mg}$ per $\mathrm{h}$ (in this case nucleated chicken erythrocyte 'ghosts' were used; for comparison, see Fig. 3). Fig. 6 shows that positively charged liposomes of phosphatidylcholine or sphingomyelin adsorbed tightly to chicken erythrocyte 'ghosts' and could not be separated by multiple washings with buffer. It is noteworthy that the respective curves that describe the degree of association of liposomes and chicken erythrocyte 'ghosts' (Fig. 6) or the rate of sphingomyelin hydrolysis (Fig. 4), both as a function of the relative proportion of hexadecylamine in the liposome, were of similar shapes.

\section{Discussion}

Hirshfeld \& Loyter (1975) showed that, after lysis in a hypo-osmotic medium, chicken erythrocyte 'ghosts' exhibited sphingomyelinase activity. The present paper shows that the enzyme, which is membrane bound, degrades not only its own sphingomyelin but also that of other membranes such as human erythrocyte 'ghosts' or liposomes. These findings and the fact that sphingomyelin is present mostly in the outer layer of the erythrocyte membrane (Colley et al., 1973) suggest that the

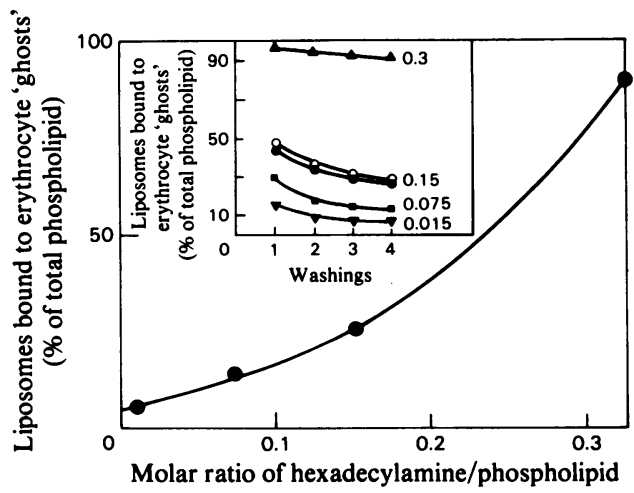

Fig. 6. Adhesion between liposomes and chicken erythrocyte 'ghosts'

Liposomes containing $\left[{ }^{14} \mathrm{C}\right]$ phosphatidylcholine or

$\left[{ }^{3} \mathrm{H}\right]$ sphingomyelin and various proportions of hexadecylamine were prepared as described in the Experimental Procedure section. Liposomal dispersion $(0.3 \mathrm{ml})$ was mixed with $0.1 \mathrm{ml}(3.8 \mathrm{mg})$ of packed sphingomyelin-depleted chicken erythrocyte 'ghosts'. After $10 \mathrm{~min}$ at $4^{\circ} \mathrm{C}, 0.7 \mathrm{ml}$ of $25 \mathrm{mM}$ Tricine $/ 9 \mathrm{mM}-\mathrm{MgCl}_{2}$ were added, and the mixture was centrifuged and washed three times successively with a buffer containing $25 \mathrm{~mm}$-Tricine, $\mathrm{pH} 7.4$, and $6 \mathrm{~mm}-\mathrm{MgCl}_{2}$. Each resulting supernatant was mixed with 2 vol. of chloroform/methanol $(1: 1, v / v)$, and the lower phase obtained was evaporated and counted for radioactivity. The remaining pellet was also extracted with chloroform $/$ methanol and the radioactivity in the extract was determined. The curve describes radioactivity remaining in a washed pellet as a function of the molar ratio of hexadecylamine to phosphatidylcholine. The inset shows the radioactivity remaining in a pellet as a function of the number of washings. The numbers represent the respective molar ratios of hexadecylamine to phosphatidylcholine. The curve described by open circles shows the results of an experiment where sphingomyelin liposomes were used instead of phosphatidylcholine.

sphingomyelinase in chicken erythrocytes might be located in the outer layer of the chicken erythrocyte membrane.

The finding that the enzyme in one membranous vesicle (chicken erythrocyte 'ghost') hydrolyses sphingomyelin in an adjacent vesicle (human erythrocyte 'ghost' or a liposome) poses difficulties in demonstrating unequivocally a true intramembrane hydrolysis of the substrate by enzyme molecules residing in the same membrane (for discussion and experimental procedures to prove this, see Rousseau \& Gatt, 1979). Existence of an intramembrane as well as intermembrane hydrolysis of sphingomyelin, though not proven unequivocally, 
is suggested by the following two observations. (a) The initial rate of hydrolysis of sphingomyelin in the chicken erythrocyte 'ghost' is greater, by one order of magnitude, than that in the external membrane (i.e. the human erythrocyte 'ghost'; see Fig. 1). (b) The curves that describe the rate of hydrolysis of sphingomyelin as a function of increasing concentrations of internal substrate (in the chicken erythrocyte 'ghosts') or of external substrate (in human erythrocyte 'ghosts' or liposomes) are seemingly dissimilar; whereas the latter is probably hyperbolic (Figs. 3 and 5), the former were seemingly parabolic (Fig. 2c), although a strict mathematical analysis of these curves was not done. Parabolic shapes such as those in Fig. 2 are a result of 'substrate activation' of the enzyme in the chicken erythrocyte 'ghosts' by the sphingomyelin residing in the same membrane (a kinetic treatment of such curves may be found in Dixon \& Webb, 1966). It is of interest that in a second membranous system (hydrolysis of diacylglycerol in rat brain microsomal fraction by membranous lipase), the curves that described the rate of substrate hydrolysis were also hyperbolic and parabolic for inter- and intramembrane utilization respectively (Rousseau \& Gatt, 1979). The possibility that the hydrolysis of sphingomyelin of the human erythrocyte 'ghost' is a consequence of transfer of this lipid to the enzymecontaining membrane of the chicken erythrocyte 'ghost' does not seem likely. Transfer or exchange of phospholipids has been shown to depend substantially on the presence of specific 'exchange proteins' (Zilversmit \& Hughes, 1976; Wirtz et al., 1972). Furthermore, as far as we know, even such an exchange has not been demonstrated for chicken erythrocytes or their 'ghosts'. Fusion between the membranes of the human and chicken erythrocyte 'ghosts' could have changed the seemingly intermembrane interaction into an intramembrane utilization. Phase microscopy showed that such fusion did not occur in the present experimental conditions (A. Loyter, unpublished work).

A most interesting aspect of the present system is the apparent latency of the chicken erythrocyte sphingomyelinase. The enzyme is seemingly inactive, unless erythrocytes are treated with a hypo-osmotic buffer. The data in Figs. 1 and 2 show a very rapid hydrolysis of the membranous sphingomyelin, which occurs after hypo-osmotic treatment of the chicken erythrocytes. Were such an activation to occur in the circulation, the entire sphingomyelin of the chicken erythrocytes would be converted into ceramide [ $N$-acylsphingosine) and phosphocholine. This conversion of an amphiphilic into a neutral lipid would markedly affect the membrane structure. The latter would probably contract and form microdroplets (Coleman et al., 1970). The finding that washed chicken erythro- cytes have a high content of sphingomyelin (Gazit $e t$ al., 1975) suggests that the enzyme is indeed inoperative in the circulation.

The presence of 'latent' phospholipases was suggested for other organelles such as human erythrocytes (Paysant et al., 1970), platelets (Picket et al., 1976), mitochondria (Parce et al., 1978), bacteria (Patriarca et al., 1972) and pancreatic juice (Slotboom et al., 1978). Non-utilization of a membranous substrate by a membranous enzyme might be a consequence of the presence of an inactive form of the enzyme, as a proenzyme (Slotboom et al., 1978), which must be activated by proteolysis (Paysant et al., 1970; Picket et al., 1976; Slotboom et al., 1978). But the possibility should not be overlooked that the enzyme molecule exists in the membrane in a fully active form, but cannot utilize the membranous lipid substrate because of physical separation (e.g. each being in a separate layer), inadequate surface pressure, necessity of a high local concentration of substrate in the membrane or a cofactor (e.g. a metal ion; Allan \& Michell, 1975; Parce et al., 1978). It is not yet possible to define the mechanism relevant to latency and activation of the sphingomyelinase in the chicken erythrocytes, nor the precise molecular nature of the intramembrane or intermembrane hydrolysis of the membranous lipid substrate by the membranous enzyme. Experiments to elucidate this mechanism and to obtain direct evidence for the existence of the two types of enzyme-substrate interactions in the respective erythrocyte 'ghosts' are now required.

This work was supported by grants from the National Institutes of Health (NS02967) to S. G., the Israeli Academy of Sciences to A. L. and the Israeli Ministry of Health to S. G. and A. L.

\section{References}

Allan, D. \& Michell, R. H. (1975) Nature (London) 258, 348-349

Barenholz, Y., Gibbes, D., Litman, B. J. \& Goll, J. (1977) Biochemistry 16, 2806-2810

Bartlett, G. R. (1959) J. Biol. Chem. 234, 466-468

Cabot, M. C. \& Gatt, S. (1976) Biochim. Biophys. Acta 431, 105-115

Cabot, M. C. \& Gatt, S. (1977) Biochemistry 16, 2330-2334

Coleman, R., Finean, J. B., Knutton, S. \& Limbrick, A. R. (1970) Biochim. Biophys. Acta 219, 81-92

Colley, C. M., Zwaal, R. F. A., Roelofsen, B. \& Van Deenen, L. L. M. (1973) Biochim. Biophys. Acta 307, 74-82

Dixon, M. \& Webb, E. C. (1966) Enzymes, 2nd edn., pp. 81-84, Longmans, London

Gatt, S. (1973) Metab. Inhibitors 34, 379-387

Gatt, S. (1967) Biochem. Biophys. Res. Commun. 68, 235-241 
Gatt, S. \& Barenholz, Y. (1973) Annu. Rev. Biochem. 42, 61-91

Gatt, S., Herzl, A. \& Barenholz, Y. (1973) FEBS Lett. 30, 281-285

Gätt, S., Freysz, L. \& Mandel, P. (eds.) (1978) Enzymes of Lipid Metabolism, Plenum Press, New York

Gazit, Y., Ohad, I. \& Loyter, A. (1975) Biochim. Biophys. Acta 382, 65-72

Hirshfeld, D. \& Loyter, A. (1975) Arch. Biochem. Biophys. 67, 186-192

Lowry, O. H., Rosebrough, N. J., Farr, A. L. \& Randall, R. J. (1951) J. Biol. Chem. 193, 265-275

Parce, J. W., Cunningham, C. C. \& Waite, M. (1978) Biochemistry 17, 1634-1639

Patriarca, P., Beckerdite, S. \& Elsbach, P. (1972) Biochim. Biophys. Acta 260, 503-600

Paysant, M., Bitran, M., Wald, B. \& Polonovski, J. (1970) Bull. Soc. Chim. Biol. 52, 1257
Picket, N. C., Jesse, R. L. \& Cohen, P. (1976) Biochem. J. 160, 405-408

Record, M. \& Gatt, S. (1979) Isr. J. Med. Sci. 15, 71

Rousseau, A. \& Gatt, S. (1979) J. Biol. Chem. 254, 7741-7745

Slotboom, A. J., Van Dam Meieras, M. C. E., Jansen, E. H. J. M., Pattus, F., Verheig, H. M. \& de Haas, J. H. (1978) in Enzymes of Lipid Metabolism (Gatt, S., Freysz, L. \& Mandel, P., eds.), pp. 137-152, Plenum Press, New York

Stoffel, W. W. (1975) Methods Enzymol. 35, 533-548

Toister, Z. \& Loyter, A. (1973) J. Biol. Chem. 248, 422-432

Wirtz, K. W. A., Kamp, H. H. \& Van Deenen, L. L. M. (1972) Biochim. Biophys. Acta 274, 606-617

Zilversmit, D. B. \& Hughes, M. E. (1976) Methods Membr. Biol. 7, 211-259 